\title{
A New Approach to Dyeing of 80 : 20 Polyester/Cotton Blended Fabric Using Disperse and Reactive Dyes
}

\author{
B. Muralidharan ${ }^{1,2}$ and S. Laya ${ }^{1,2}$ \\ ${ }^{1}$ Department of Industrial Chemistry, Alagappa University, Karaikudi, TamilNadu 630003, India \\ ${ }^{2}$ Department of Chemistry, Birla Institute of Technology and Science-Pilani, \\ Dubai Campus, Dubai International Academic City, P.O. Box 345055, Dubai, UAE
}

Correspondence should be addressed to B. Muralidharan, bmdharan@gmail.com

Received 9 April 2011; Accepted 3 May 2011

Academic Editor: P. de Lima-Neto

Copyright (c) 2011 B. Muralidharan and S. Laya. This is an open access article distributed under the Creative Commons Attribution License, which permits unrestricted use, distribution, and reproduction in any medium, provided the original work is properly cited.

Polyester/Cotton blended fabrics are normally dyed by two-bath or one-bath two-step dyeing method. This paper deals with a new approach involving azeotropic ternary mixture of organic solvents pretreatment to dye polyester/cotton blends using disperse and reactive dyes in one-bath method. The effect of solvent pretreatments on dyeability, fastness, and few physicochemical properties has been investigated involving SEM, FTIR, DSC, and XRD studies, and results are presented.

\section{Introduction}

Commercially polyester/cotton blended fabrics are dyed by two-bath or one-bath two-step dyeing method employing suitable dyes and chemicals for each fiber [1].

Two bath dyeing methods are relatively long and complicated. The one-bath two-step dyeing procedure is shorter as compared to two-bath method, but the drawbacks are lower dyeability and poor reproducibility [2-4]. Dyeing of cotton by conventional reactive dyes is carried out under alkaline conditions at $80^{\circ} \mathrm{C}$, but this is entirely different condition from that of polyester dyeing, which is carried out at acidic conditions over $120^{\circ} \mathrm{C}$ by using dispersed dyes. Many research works have been carried out to dye polyester/cotton blends in one-bath dyeing method using conventional dispersed dyes and newly developed reactive dyes which can be dyed at acidic or neutral conditions around $100-130^{\circ} \mathrm{C}$ and are added simultaneously to the same bath [5-8].

Youssef et al. [9] have developed a dyeing method for polyester/cotton blend fabrics using sodium edetate as an alkaline buffering agent. Selected mono- and bifunctional reactive dyes were used in combination with the alkali stable disperse dyes for dyeing of polyester/cotton blend. One-bath method for dyeing of polyester/cotton blends with reactive disperse dyes using supercritical carbon dioxide as a solvent at $393 \mathrm{~K}$ and $20 \mathrm{MPa}$ was successfully investigated by Shingo et al. [10]. A very recent research work reported by Najafi et al. [11] discussed the process of dyeing polyester/cotton fabrics using disperse/reactive dyestuff in one-bath dyeing process after coating the fabric with chitin biopolymer and then dyed with sulphato ethyl sulphonyl disperse/reactive dyes. Reports are available on the use of single solvent assisted dyeing of polyester and polyester/cotton blends [12-14]. Dystar Textilfarben GMBH \& Co. has patented dyeing of polyester/cotton blends fabrics with disperse and reactive dyes in a one-bath process in alkaline medium using disperse dyes which are stable in alkaline medium [15].

The present work was undertaken to establish a new method for dyeing polyester/cotton blends by pretreating the fibre blend using nonaqueous azeotropic solvent mixture to get improved dyeing results. The term Azeotrope (Greek) means "to boil unchanged", that is, the vapour boiling from a liquid has the same composition as the liquid. The composition of the ternary mixtures was fixed by referring to azeotropic data published by Ryland [16] and Lecat [17]. The polyester/cotton blend of $80: 20$ composition was treated 
<smiles>CCN(CCC#N)c1ccc(N=Nc2cc(Cl)c(S(N)(=O)=O)cc2Cl)cc1</smiles><smiles>CCCCCNc1ccc(N=Nc2c(Br)cc([N+](=O)[O-])cc2C#N)c(NC(=O)CC)c1</smiles>

(c)

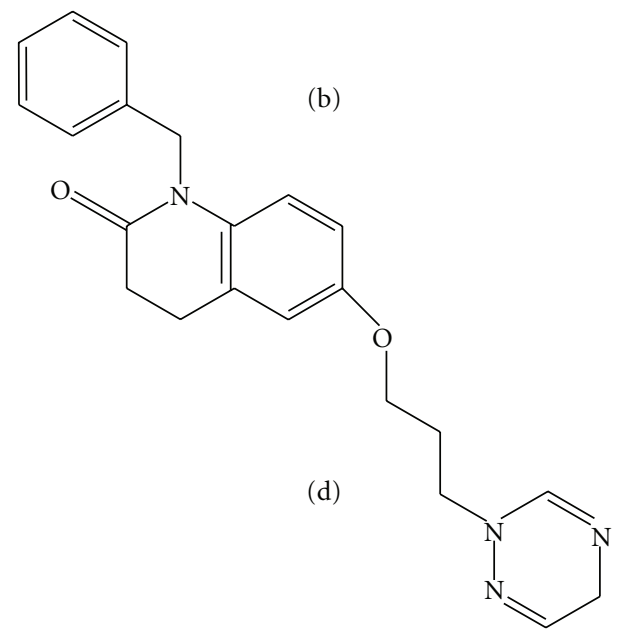

Scheme 1: (a) Foron Brilliant Red E-2BL200 F (C.I. Disperse Red 60); (b) Foron Brilliant Orange S-FL (C.I. Disperse Orange 96); (c) Foron Blue SE 2R (Disperse Blue183); (d) Foron Brilliant Yellow E-3GFL (C.I. Disperse Yellow 49).

with two different azeotropic solvent mixtures for various durations and their physical, structural, chemical, and dyeing behavior was analyzed.

\section{Experimental}

2.1. Materials, Dyes, and Chemicals. 80:20 polyester/cotton fabric (80:20 PCF):

A plain woven polyester/cotton fabric of the following specification was used.

(i) Types of yarn-filament.

(ii) Ends per inch-146.

(iii) Picks per inch-82.

2.1.1. Dyes. The following four disperse dyes (Scheme 1) were used for dyeing the polyester component of the blend as supplied by the manufacturer (Parishi Chemicals, Surat, India) without further purification.

The following four reactive dyes (Scheme 2) were used for dyeing the cotton component of the blend as supplied by the manufacturer (Ridhi Sidhi Trading Co., Mumbai, India) without further purification.

2.1.2. Chemicals. The details of Azeotropic mixtures of organic solvents used are shown in Table 1. Commercially available anionic wetting agent, dispersing agent, Glauber's salt, sodium bicarbonate (to maintain the $\mathrm{pH}$ between 10 and 11), and borax as buffering agent were used in the dyebath. Ladipur MCL was used for reduction clearing of the fabric after dying. All chemicals used were Fischer-LR Grade.

2.1.3. Apparatus. Padding mangle was used to squeeze the pretreated fabric to aid the penetration of solvent mixture into the interior of the fibre samples. Dyeing was performed using the Rota-dyer bath (Rota dyer $18 \times 100$-N machine, R.B. Electronics \& Engineering Pvt. Ltd., Mumbai-53, India).

2.1.4. Pretreatments. The above-said azeotropic mixtures (Table 1) were used as pretreater. The pretreatments were carried out at room temperature for various time intervals, namely, 2, 4, 6, 8, 10, 20, and 30 minutes. The pretreated fabrics were then squeezed in the padding mangle and then air dried for removal of residual solvent mixture. Then, the fabrics were subjected to dyeing.

\subsubsection{Dyeing Recipe.}

(i) Disperse dye: $2 \%$.

(ii) Reactive dye: $2 \%$.

(iii) Glauber's salt: 5 gpl.

(iv) Soda ash: 3 gpl.

(v) Borax: 5 gpl.

(vi) $\mathrm{pH}$ of the dye bath: 10 to 11 .

(vii) MLR (material to liquor ratio) $: 1: 50$.

(viii) Temperature: 80,95 , and $110^{\circ} \mathrm{C}$.

(ix) Time: 30, 45, and 60 minutes. 


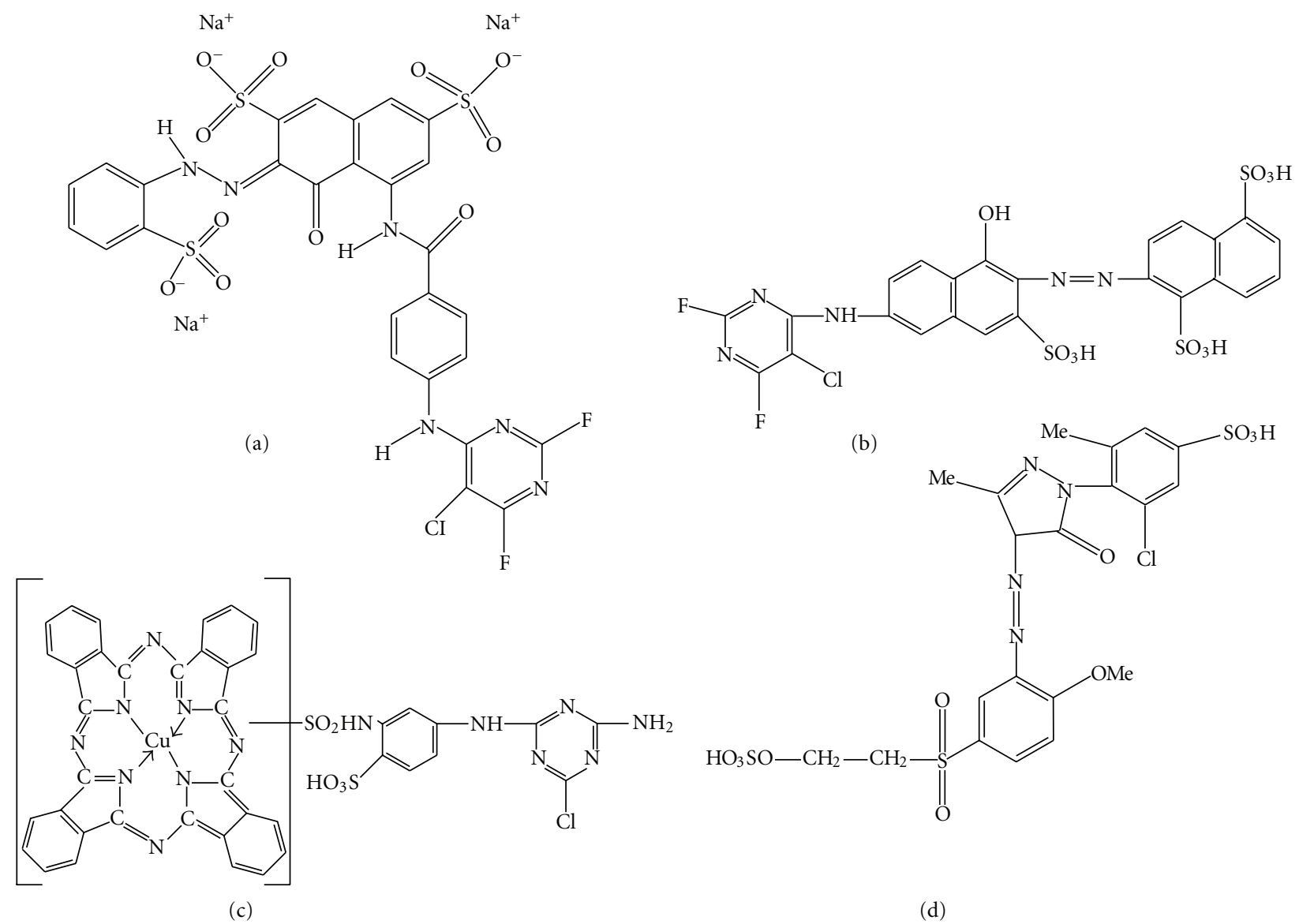

Scheme 2: (a) Drimarene Brilliant Red K-BL (C.I. Reactive Red 124); (b) Drimarene Brilliant Orange (C.I. Reactive Orange 64); (c) Drimarene Turquoise K-2B (C.I. Reactive Blue 116); (d) C.I. Reactive Yellow 14.

TABLE 1: Details of azeotropic solvent mixture.

\begin{tabular}{|c|c|c|c|c|c|c|}
\hline S. no & Solvent System & Weight $\%$ & Volume \% & $\begin{array}{l}\text { Solubility } \\
\text { parameter }\end{array}$ & $\begin{array}{c}\text { Polarity } \\
\text { Index }\end{array}$ & B. P. $\left({ }^{\circ} \mathrm{C}\right)$ \\
\hline & Acetone & 24.3 & 30.6 & 10.0 & & \\
\hline \multirow[t]{3}{*}{1} & Ethyl alcohol & 10.4 & 13.2 & 12.7 & 14.5 & 63.2 \\
\hline & Chloroform (Ac-EA-Cf) & 65.3 & 44.0 & 9.3 & & \\
\hline & Acetone & 51.1 & 64.3 & 10.0 & & \\
\hline \multirow[t]{2}{*}{2} & Methyl acetate & 5.6 & 6.0 & 9.8 & 9.5 & 49.7 \\
\hline & $\mathrm{n}$-Hexane (Ac-MAc-nH) & 43.3 & 66.1 & 7.3 & & \\
\hline
\end{tabular}

The pretreated samples were introduced into the abovesaid dye bath and kept under these conditions for 10 minutes. Then, calculated amount of dye solution and chemicals were added into the dye bath and then required temperature was reached by increasing the bath temperature at a rate of $2^{\circ} \mathrm{C} / \mathrm{min}$. Dyeings were carried out for the above-mentioned durations. After the completion of the dyeing time, the temperature was brought down to room temperature gradually, then the dyed fabrics were taken out and washed with water. The fabrics after dyeing and washings were reduction cleared by using commercially available reduction clearing agent, Ladipur MCL (Clariant Chemicals, India). Then, it was washed with water at room temperature and then dried in a hot air oven.

2.2. Measurement of Dye Uptake. The amount of dye pickup of polyester/cotton fabrics during dyeing was determined spectrophotometrically using spectrophotometer (Labomedmodel spectro 23 RS, USA).

2.3. Test for Colour Fastness. The untreated and solventpretreated 80:20 PCFs after dyeing were tested for their 
wash fastness, light fastness and rub fastness using AATCC test methods (AATCC technical manual 2000). The washing fastness, was evaluated by AATCC method 61(2A) using an Atlas-Launder Ometer. Fastness to light was evaluated by AATCC method 16E using an Atlas CI $3000+$ Xenon Weatherometer. The fastness to rubbing was also evaluated as per AATCC 116-1995 standards using crock meter.

2.4. Determination of Weight Loss. The weight loss percentage of the treated fabrics was determined by measuring the weights before and after pretreatments using an electronic balance Sartorius-GD 503-Germany.

2.5. Abrasion Resistance. The abrasion resistance of the fabric samples after and before solvent treatment was measured by Martindale abrasion tester as per ASTM D4966 test method.

2.6. SEM Topography. Scanning electron microscopic studies were made on treated and untreated samples with S-3000HHitachi, Japan to study surface modifications if any caused by the solvent pretreatments using azeotropic mixtures. The samples were imaged with a magnification of 500x for better understanding of the inner core of the sample.

2.7. FTIR Analysis. Fourier Transform infrared spectral analysis of the treated and untreated fabrics was recorded in the range of $4000-400 \mathrm{~cm}^{-1}$ using Perkin Elmer spectrometer (spectrum BX, USA) with built-in spectral matching computerized software. The fabric samples were made into individual fibers and were mounted onto the instrument for recording the spectrum.

2.8. Thermal Analysis. Thermal analysis of the untreated and solvent-mixture-pretreated 80:20 PCF was made using Perkin Elmer Pyris 6, USA at a temperature range of -50 to $400^{\circ} \mathrm{C}$ with a heating rate of $50^{\circ} \mathrm{C} / \mathrm{min}$ under inert atmosphere of nitrogen gas at a rate of $20 \mathrm{~mL} / \mathrm{min}$ [18].

2.9. X-ray Diffraction Studies. X-Ray diffraction studies using PANalytival-mode X'Pert PRO was carried out for both untreated and solvent-treated 80:20 PCFs for determining the crystalline and amorphous region of both treated and untreated samples. The samples were analyzed by observing number of counts as a function of scattering angle $(2 \theta)$.

\section{Results and Discussions}

3.1. Dyeing Behavior of Fabric. The effect of azeotropic mixture of solvent pretreatments on the dyeing behavior of 80:20 PCF was studied by dyeing the pretreated and untreated fabrics for different dyeing time intervals $(30,45$, and 60 minutes) and at different temperatures (80, 95, and $\left.110^{\circ} \mathrm{C}\right)$. The dye uptake results are presented in the Figures 1 , $2,3,4,5,6,7$, and 8 . It is clear from the figures that maximum dye uptake is observed in the case of samples pretreated for 8 minutes with the solvent systems Ac-EA-Cf and Ac-MAc-nH. As the pretreatment time increases, the dye uptake is found to increase with increase in dyeing temperature and duration of dyeing. The dye uptake for the samples treated beyond

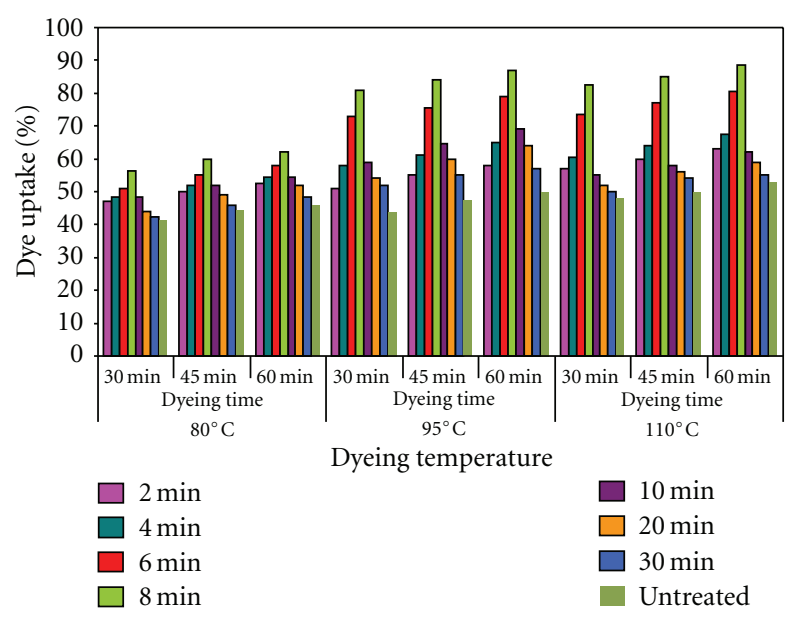

Figure 1: Dye uptake of $80: 20$ PCF treated with Ac-EA-Cf (Disperse Red 60 and Reactive Red 124).

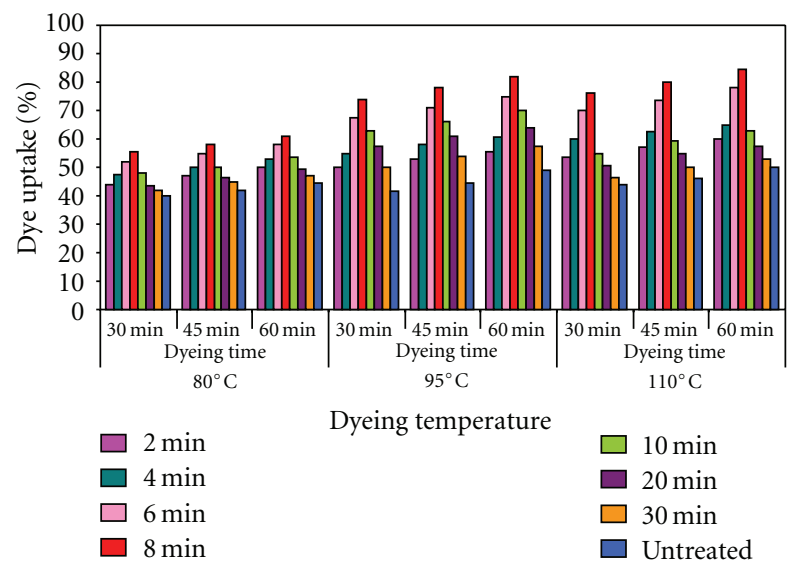

Figure 2: Dye uptake of $80: 20$ PCF treated with Ac-EA-Cf (Disperse Orange 96 and Reactive Orange 64).

$8 \mathrm{~min}$ was found decreasing. The change in dyeing behavior of the treated fabrics reflects changes in fibre structure of the treated fabrics caused by azeotropic mixtures of solvents. Due to solvent pretreatment, the molecular structure of the fabrics gets loosened, resulting in increased dye uptake. The improvement in the dye uptake of treated samples is probably due to the large increase in inter surface by swelling or plasticizing action, greater segmental mobility of polymer molecules, formation of micro voids, and so on [19-21]. The pretreatment enabled to get better dye uptake even at a low temperature of $80^{\circ} \mathrm{C}$, and in the cases where the pretreatment time is above 8 minutes, the dye uptake is found to decrease which may be due to the desorption of dye from the fabric due to irreversible swelling of the fibre. The extent of improvement in dyeing behavior was found to be different for different dyes.

3.2. Fastness Properties. Tables 2, 3, and 4 show the wash, light, and rubbing fastness properties of the treated and untreated 80:20 PCF. The results indicate that the solvent treatments involving azeotropic mixtures of solvents 


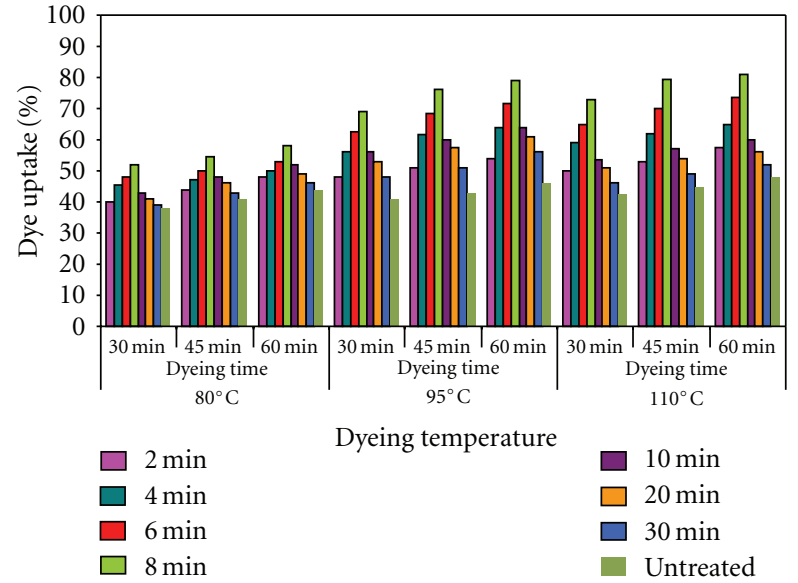

Figure 3: Dye uptake of $80: 20$ PCF treated with Ac-EA-Cf (Disperse Blue 183 and Reactive Blue 116).

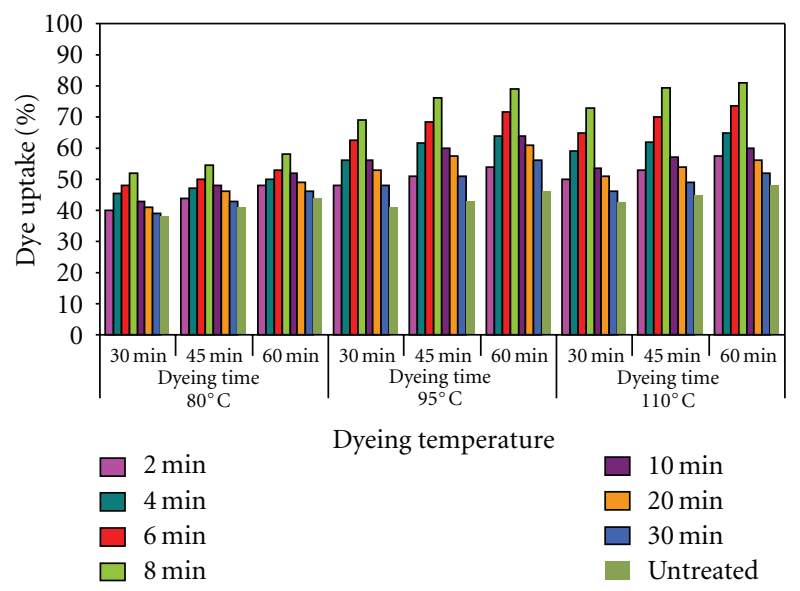

Figure 4: Dye uptake of $80: 20$ PCF treated with Ac-EA-Cf (Disperse Yellow 49 and Reactive Yellow 14).

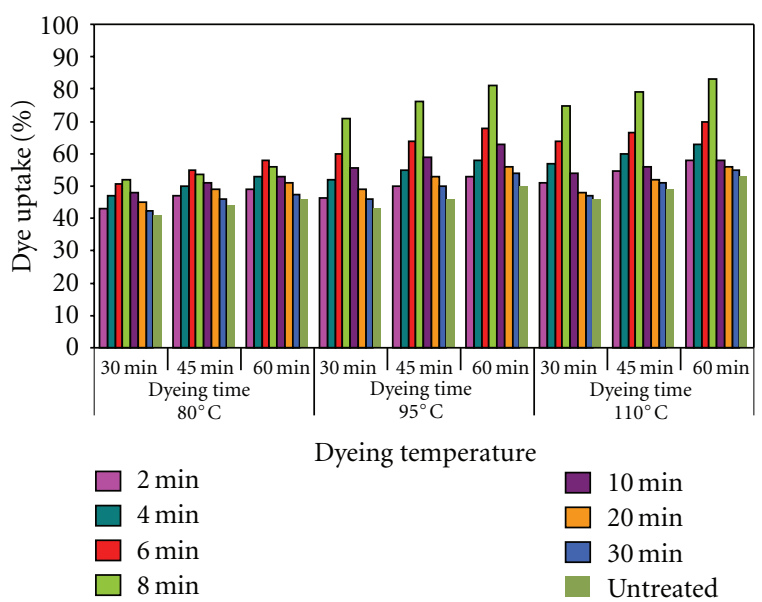

Figure 5: Dye uptake of $80: 20$ PCF treated with Ac-MAc-nH (Disperse Red 60 and Reactive Red 124).

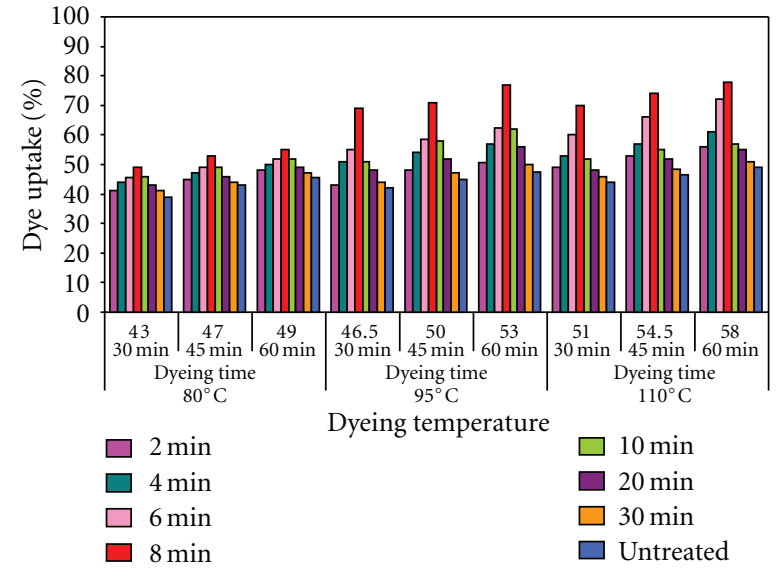

Figure 6: Dye uptake of $80: 20$ PCF treated with Ac-MAc-nH (Disperse Orange 96 and Reactive Orange 64).

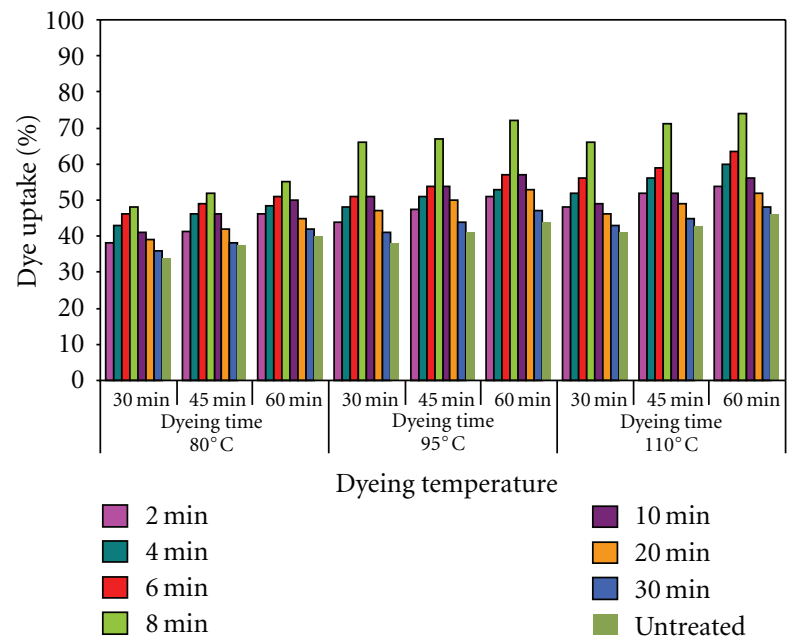

Figure 7: Dye uptake of $80: 20$ PCF treated with Ac-MAc-nH (Disperse Blue 183 and Reactive Blue 116).

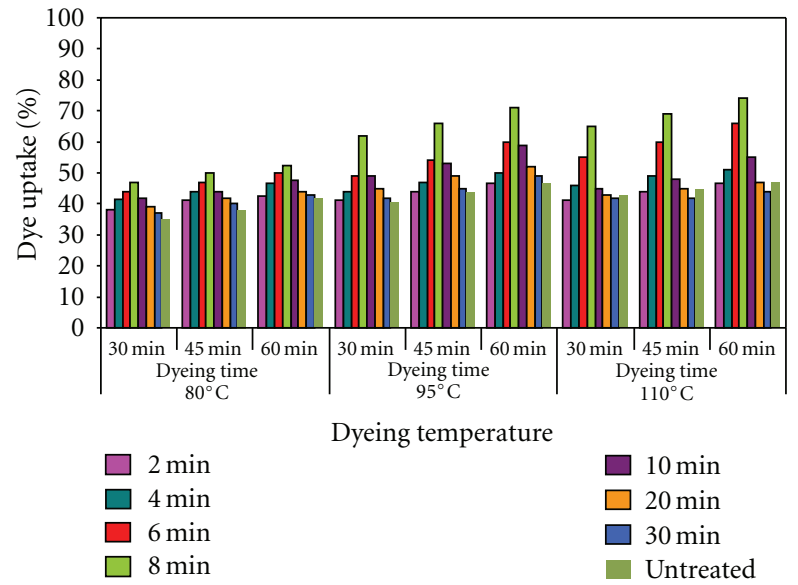

FIgure 8: Dye uptake of $80: 20$ PCF treated with Ac-MAc-nH (Disperse Yellow 49 and Reactive Yellow 14). 
have slightly improved the fastness properties of the dyed polyester/cotton blended fabrics. This may be due to the fact that the solvent pretreatments have improved the penetration of the dyestuff molecules into the interior of the fiber matrix and have improved the stability of dye-fiber bond.

\subsection{Weight Loss and Abrasion Resistance Measurements.}

Table 5 shows the changes in weight and abrasion resistance of the solvent pretreated fabrics in comparison with untreated fabrics. It was found that the weight loss is very small and is dependent upon the pretreatment time. As the pretreatment time increases, there was an increase in weight loss. The abrasion resistance measurements of the treated materials show that there was a slight increase in abrasion resistance. The extent of increase in abrasion resistance was found to increase with increase in treatment duration due to increased pitting of fiber surface. However, the overall effect of solvent pretreatment has not caused any detrimental effect.

3.4. Tearing Strength Measurements. Tearing strength measurement of untreated and azeotropic solvent-mixturepretreated samples showed that there is significant improvement in the tearing strength of the treated materials. In all the cases, the maximum load applied has been found to increase and the elongation percentage remains almost constant. The above changes may be due to very less influence of the solvent treatment on crystallinity index. The mechanical properties of textile fibers depend not only on the degree of crystallinity of fibers but also on the various secondary valence forces that operate in the polymer. The improvement in the strength of treated materials can be attributed to improvement in the structural order of the polymer matrix and generation of more number of crystallites, leading to improvement in the resistance power to deform the material with higher interchain bond. These observations are strengthened by the $\mathrm{XRD}$ and DSC results as well. The present observations are in conformity with the reports available on the effect of solvent pretreatment on polymers where, in the solvents, it does not penetrate the compact crystalline region in the polymer and therefore do not affect the strength of the polymer material [22-24].

3.5. Scanning Electron Microscopy Studies. Scanning electron-micrographs of untreated and solvent-pretreated $80: 20$ PCF are presented in Figures 9, 10, and 11. The untreated samples exhibit smooth surface texture. In the treated samples, it appears that the solvent mixture attacked almost the entire surface of the fiber compared to untreated samples. As the duration of pretreatment increased, there was progress in attack and erosion propagates inside the fiber resulting in the formation of elongated pits or cavities on the surface. This is also supported by the fact that the dye uptake of solvent pretreated fabric materials has improved because of development of voids. The observed results resemble those of earlier reports available in the literature $[13,25]$.

3.6. FTIR Studies. The FTIR spectrum of $80: 20$ PCF before and after solvent treatment is shown in Figures 12 and 13.
FTIR was recorded to assess structural change if any made in the fiber of the alteration of existing functional groups as a consequence of azeotropic solvent mixture pretreatments. It was found, from the spectra, that the patterns are almost identical for both treated and untreated samples without any additional peaks. However, on comparing the samples treated with the two different azeotropic solvent mixtures, Ac-EA-Cf caused a slight shift in the position of the peak to a higher wave number than that treated with Ac-MAc-nH due to its higher polarity index. The extent of shift was found to be dependent on solvent pretreatment time. A broad peak at $1730 \mathrm{~cm}^{-1}$ is characteristic of carbonyl stretching of $\alpha-\beta$ unsaturated ester. In the case of solvent treated fiber, the width of the peak had reduced and the peak value has been shifted to higher wave number, that is, $1750 \mathrm{~cm}^{-1}$. A small peak in the region between 800 and $850 \mathrm{~cm}^{-1}$ can be accounted for out-of-plane bending of aromatic ring system. The peak at $1250 \mathrm{~cm}^{-1}$ and $1300 \mathrm{~cm}^{-1}$ may be due to $\mathrm{C}-\mathrm{O}$ stretching of the polymer back bone. An intense peak at $2350-2360 \mathrm{~cm}^{-1}$ can be attributed to methylene C$\mathrm{H}$ stretching. The small peak close to $3000 \mathrm{~cm}^{-1}$ can be correlated to $\mathrm{C}-\mathrm{H}$ stretching of aromatic ring. An interesting feature in the above-discussed spectrum was that an additional sharp small peak observed at around $3600 \mathrm{~cm}^{-1}$ corresponds to free $-\mathrm{OH}$ groups of cellulose component indicating that solvent treatment had increased the extent of amorphous region in the cotton component of the material. This trend was further been supported by the results of strength measurements and SEM studies. The observed small peaks between the regions $1110-1150 \mathrm{~cm}^{-1}$ were due to cellulosic component of the fiber materials $[26,27]$.

3.7. Thermal Studies. DSC curves of $80: 20$ PCF treated with azeotropic solvents mixtures and untreated samples are shown in Figures 14 and 15. In each case, the starting temperature and peak melting temperature (Table 6) are noted. The final melting temperature corresponds to the melting of the most stable crystallite whereas the peak melting temperature is taken as the temperature at the maximum of melting endotherm [28]. The starting temperature is the starting of the melting endotherm and can be regarded as the melting of the smallest crystallite in the sample. The DSC thermograms of solvent pretreated samples obtained are found to be almost identical with that of untreated samples with small changes in terms of starting temperature, peak temperature, and melting temperature. However, maximum heat flow has increased considerably for solvent pretreated samples due to solvent-induced crystallization. During the interaction of the polymer with the solvent, the solvent enters into the amorphous region of polymer structure, weakens polymer-polymer interaction, replaces it with polymersolvent interaction, induces extensive segmental motion, and lowers the effective glass transition temperature of material. The polymer chains rearrange themselves into a lower free energy state. This induces crystallization even in the swollen state. The interaction of solvent with the polymer may be of two types, namely, intercrystalline interaction and intracrystalline interaction. In the case of intercrystalline interaction, the solvent penetrates inside the amorphous 
TABLE 2: Wash fastness properties of treated and untreated $80: 20$ PCF.

\begin{tabular}{|c|c|c|c|c|c|c|c|c|c|c|}
\hline \multirow[t]{2}{*}{$\begin{array}{l}\text { Solvent } \\
\text { system }\end{array}$} & \multirow[t]{2}{*}{ Dye } & \multirow[t]{2}{*}{ Dyeing temp. $\left({ }^{\circ} \mathrm{C}\right)$} & \multirow[t]{2}{*}{ Untreated } & \multicolumn{7}{|c|}{$\begin{array}{c}\text { Grading } \\
\text { Pretreatment time (min.) }\end{array}$} \\
\hline & & & & 2 & 4 & 6 & 8 & 10 & 20 & 30 \\
\hline \multirow{12}{*}{ Ac-EA-Cf } & \multirow{3}{*}{ A } & 80 & 3 & 3 & $3-4$ & $4-5$ & $4-5$ & 4 & 4 & 4 \\
\hline & & 95 & 3 & 4 & $4-5$ & 5 & 5 & $4-5$ & 4 & 4 \\
\hline & & 110 & 4 & 4 & $4-5$ & 5 & 5 & $4-5$ & $4-5$ & 4 \\
\hline & \multirow{3}{*}{ B } & 80 & 3 & 3 & $3-4$ & $4-5$ & $4-5$ & 4 & 4 & 4 \\
\hline & & 90 & 3 & 4 & $4-5$ & 5 & 5 & $4-5$ & 4 & 4 \\
\hline & & 110 & 4 & 4 & $4-5$ & 5 & 5 & $4-5$ & 4 & 4 \\
\hline & \multirow{3}{*}{$\mathrm{C}$} & 80 & 3 & 3 & $3-4$ & $4-5$ & $4-5$ & 4 & 4 & 4 \\
\hline & & 95 & 4 & 4 & $4-5$ & 5 & 5 & $4-5$ & 4 & 4 \\
\hline & & 110 & 4 & 4 & $4-5$ & 5 & 5 & $4-5$ & $4-5$ & 4 \\
\hline & \multirow{3}{*}{$\mathrm{D}$} & 80 & 3 & 3 & $3-4$ & $4-5$ & $4-5$ & 4 & 4 & 4 \\
\hline & & 95 & 3 & 4 & $4-5$ & 5 & 5 & $4-5$ & 4 & 4 \\
\hline & & 110 & 4 & 4 & $4-5$ & 5 & 5 & $4-5$ & 4 & 4 \\
\hline \multirow{12}{*}{ Ac-MAc-Nh } & \multirow{3}{*}{ A } & 80 & 3 & 3 & $3-4$ & $4-5$ & $4-5$ & 4 & 4 & 4 \\
\hline & & 95 & 3 & 4 & $4-5$ & 5 & 5 & $4-5$ & 4 & 4 \\
\hline & & 110 & 4 & 4 & $4-5$ & 5 & 5 & $4-5$ & 4 & 4 \\
\hline & \multirow{3}{*}{ B } & 80 & 3 & 3 & $3-4$ & $4-5$ & $4-5$ & 4 & 4 & 4 \\
\hline & & 95 & 3 & 4 & $4-5$ & 5 & 5 & 4-5 & 4 & 4 \\
\hline & & 110 & 4 & 4 & $4-5$ & 5 & 5 & 4 & 4 & 4 \\
\hline & \multirow{3}{*}{ C } & 80 & 3 & 3 & $3-4$ & $4-5$ & $4-5$ & 4 & 4 & 4 \\
\hline & & 95 & 3 & 4 & $4-5$ & 5 & 5 & $4-5$ & 4 & 4 \\
\hline & & 110 & 4 & 4 & $4-5$ & 5 & 5 & $4-5$ & 4 & 4 \\
\hline & \multirow{3}{*}{ D } & 80 & 3 & 3 & $3-4$ & $4-5$ & $4-5$ & $4-5$ & 4 & 4 \\
\hline & & 95 & 3 & 4 & $4-5$ & 5 & 5 & 4 & 4 & 4 \\
\hline & & 110 & 4 & 4 & $4-5$ & 5 & 5 & $4-5$ & 4 & 4 \\
\hline
\end{tabular}

A: Disperse Red 60 and Reactive Red 124; B: Disperse Orange 96 and Reactive Orange 64; C: Disperse Blue 183 and Reactive Blue 116; D: Disperse Yellow 49 and Reactive Yellow 14.

region only. The polymer chains within this region are under lower stress, and this generally results in the rearrangement of molecular chains $[29,30]$. In this case, crystallization takes place in the swollen state and crystalline areas of the sample increase. On the other hand, in the case of intracrystalline interaction, the interacting solvent penetrates inside the crystalline region, decrystallizes the sample, and affects higher lateral order parts of the fiber. In the present study, the interaction of solvent with the fiber material is found to be intercrystalline interaction. This is evident from the considerable increase in the melting heat for solventtreated samples due to solvent-induced crystallization. It is further supported by the observed small increase in starting temperature, peak temperature, and melting temperature of the treated samples.

3.8. XRD Studies of 80:20 PCF. X-ray diffraction studies on a polymer are mainly concerned, with study of crystalline, amorphous, and semicrystalline regions/phases, which are responsible for observing their respective electrical and mechanical properties. X-ray diffraction pattern of most polymers contains sharp as well as broad and diffuse peak. The sharp peak corresponds to crystalline regions; the diffuse and the broad ones refer to amorphous region [31-34]. The interaction of solvent with polymer results in recrystallization and decrystallization of the corresponding polymer 
TABLE 3: Light fastness properties of treated and untreated $80: 20$ PCF.

\begin{tabular}{|c|c|c|c|c|c|c|c|c|c|c|}
\hline \multirow[t]{2}{*}{$\begin{array}{l}\text { Solvent } \\
\text { system }\end{array}$} & \multirow[t]{2}{*}{ Dye } & \multirow[t]{2}{*}{ Dyeing temp. $\left({ }^{\circ} \mathrm{C}\right)$} & \multirow[t]{2}{*}{ Untreated } & \multicolumn{7}{|c|}{$\begin{array}{l}\text { Grading } \\
\text { Pretreatment time (min.) }\end{array}$} \\
\hline & & & & 2 & 4 & 6 & 8 & 10 & 20 & 30 \\
\hline \multirow{12}{*}{ Ac-EA-Cf } & \multirow{3}{*}{$\mathrm{A}$} & 80 & 5 & 5 & $5-6$ & $6-7$ & 7 & $6-7$ & 6 & 6 \\
\hline & & 95 & $5-6$ & $5-6$ & $5-6$ & 7 & 7 & $6-7$ & 6 & 6 \\
\hline & & 110 & $6-7$ & $6-7$ & $6-7$ & 7 & 7 & $6-7$ & 6 & 6 \\
\hline & \multirow{3}{*}{$\mathrm{B}$} & 80 & 5 & 5 & $5-6$ & $6-7$ & 7 & $6-7$ & 6 & 6 \\
\hline & & 90 & 5 & 5 & 6 & 7 & 7 & $6-7$ & $6-7$ & 6 \\
\hline & & 110 & $6-7$ & $6-7$ & 6 & 7 & 7 & $6-7$ & $6-7$ & 6 \\
\hline & \multirow{3}{*}{$\mathrm{C}$} & 80 & 5 & 5 & $5-6$ & 6 & 6 & 6 & $5-6$ & $5-6$ \\
\hline & & 95 & 5 & 5 & 6 & $6-7$ & 7 & $6-7$ & 6 & 6 \\
\hline & & 110 & 6 & 6 & 6 & $6-7$ & 7 & $6-7$ & 6 & 6 \\
\hline & \multirow{3}{*}{$\mathrm{D}$} & 80 & 5 & 5 & $5-6$ & 6 & 6 & 6 & 6 & $5-6$ \\
\hline & & 95 & 5 & 5 & 6 & $6-7$ & 6 & $6-7$ & 6 & 6 \\
\hline & & 110 & 6 & 6 & 6 & $6-7$ & 7 & $6-7$ & 6 & 6 \\
\hline \multirow{12}{*}{ Ac-MAc-Nh } & \multirow{3}{*}{ A } & 80 & 5 & 5 & $5-6$ & $6-7$ & $6-7$ & 6 & 6 & 6 \\
\hline & & 95 & $5-6$ & 5 & $5-6$ & 7 & 7 & $6-7$ & 6 & 6 \\
\hline & & 110 & 6 & 6 & 6 & 7 & 7 & $6-7$ & 6 & 6 \\
\hline & \multirow{3}{*}{ B } & 80 & $4-5$ & 5 & $5-6$ & $6-7$ & $6-7$ & 6 & 6 & 6 \\
\hline & & 95 & $5-6$ & $5-6$ & 6 & 7 & 7 & $6-7$ & 6 & 6 \\
\hline & & 110 & 6 & 6 & $6-7$ & 7 & 7 & $6-7$ & 6 & 6 \\
\hline & \multirow{3}{*}{$\mathrm{C}$} & 80 & 4 & $4-5$ & $5-6$ & 6 & $6-7$ & 6 & 6 & 6 \\
\hline & & 95 & $5-6$ & 6 & 6 & 7 & 7 & $6-7$ & 6 & 6 \\
\hline & & 110 & 6 & 6 & 6 & 7 & 7 & $6-7$ & 6 & 6 \\
\hline & \multirow{3}{*}{$\mathrm{D}$} & 80 & 4 & 4 & 5 & 6 & $6-7$ & 6 & 6 & 6 \\
\hline & & 95 & $5-6$ & $5-6$ & 6 & 7 & $6-7$ & $6-7$ & 6 & 6 \\
\hline & & 110 & $5-6$ & $5-6$ & 6 & 7 & 7 & $6-7$ & 6 & 6 \\
\hline
\end{tabular}

A: Disperse Red 60 and Reactive Red 124; B: Disperse Orange 96 and Reactive Orange 64; C: Disperse Blue 183 and Reactive Blue 116; D: Disperse Yellow 49 and Reactive Yellow 14.

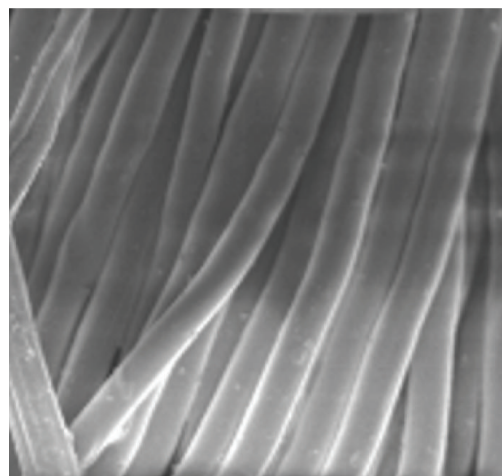

FIGURE 9: SEM photograph of untreated 80:20 PCF.

contents. XRD patterns were recorded for the untreated and pretreated $80: 20$ PCF to evaluate the effect of pretreatments and are presented in Figures 16 and 17. The results from XRD

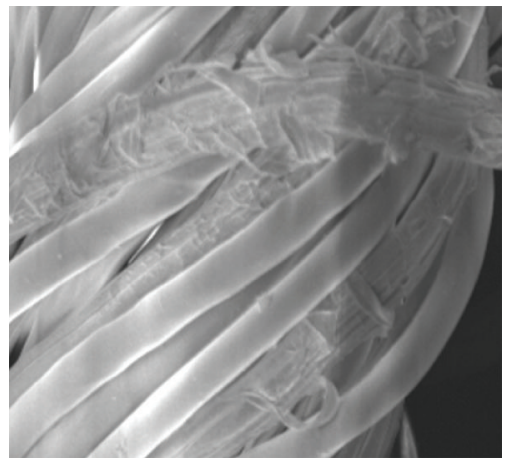

Figure 10: SEM photograph of AcEA-Cf-treated 80:20 PCF.

reveals that the solvent treatment disturbs the amorphous region of the fabric material used in the present study, probably creates more cavity and pores resulting in the 
TABLE 4: Rubbing fastness properties of treated and untreated $80: 20$ PCF.

\begin{tabular}{|c|c|c|c|c|c|c|c|c|c|c|}
\hline \multirow[t]{2}{*}{$\begin{array}{l}\text { Solvent } \\
\text { system }\end{array}$} & \multirow[t]{2}{*}{ Dye } & \multirow[t]{2}{*}{ Dyeing temp. $\left({ }^{\circ} \mathrm{C}\right)$} & \multirow[t]{2}{*}{ Untreated } & \multicolumn{7}{|c|}{$\begin{array}{c}\text { Grading } \\
\text { Pretreatment time (min.) }\end{array}$} \\
\hline & & & & 2 & 4 & 6 & 8 & 10 & 20 & 30 \\
\hline \multirow{12}{*}{ Ac-EA-Cf } & \multirow{3}{*}{ A } & 80 & $2-3$ & 3 & 3 & 4 & 4 & 4 & 4 & 4 \\
\hline & & 95 & $3-4$ & 4 & 4 & 5 & 5 & $4-5$ & $4-5$ & $4-5$ \\
\hline & & 110 & 4 & 4 & $4-5$ & 5 & 5 & 5 & 5 & 5 \\
\hline & \multirow{3}{*}{ B } & 80 & $2-3$ & 3 & 3 & 4 & 4 & 4 & 4 & 4 \\
\hline & & 90 & $3-4$ & 4 & 4 & 5 & 5 & $4-5$ & $4-5$ & $4-5$ \\
\hline & & 110 & 4 & 4 & $4-5$ & 5 & 5 & 5 & 5 & 5 \\
\hline & \multirow{3}{*}{$\mathrm{C}$} & 80 & $2-3$ & 3 & 3 & 4 & 4 & 4 & 4 & 4 \\
\hline & & 95 & $3-4$ & 4 & 4 & 5 & 5 & $4-5$ & $4-5$ & $4-5$ \\
\hline & & 110 & 4 & 4 & $4-5$ & 5 & 5 & 5 & 5 & 5 \\
\hline & \multirow{3}{*}{$\mathrm{D}$} & 80 & $2-3$ & 3 & 3 & 4 & 4 & 4 & 4 & 4 \\
\hline & & 95 & $3-4$ & 4 & 4 & 5 & 5 & $4-5$ & $4-5$ & 4-5 \\
\hline & & 110 & 4 & 4 & $4-5$ & 5 & 5 & 5 & 5 & 5 \\
\hline \multirow{12}{*}{ Ac-MAc-Nh } & \multirow{3}{*}{ A } & 80 & $2-3$ & 3 & 3 & 4 & 4 & 4 & 4 & 4 \\
\hline & & 95 & $3-4$ & 4 & 4 & 5 & 5 & 4-5 & $4-5$ & $4-5$ \\
\hline & & 110 & 4 & 4 & $4-5$ & 5 & 5 & 5 & 5 & 5 \\
\hline & \multirow{3}{*}{ B } & 80 & $2-3$ & 3 & 3 & 4 & 4 & 4 & 4 & 4 \\
\hline & & 95 & $3-4$ & 4 & 4 & 5 & 5 & 4-5 & 4-5 & 4-5 \\
\hline & & 110 & 4 & 4 & 4-5 & 5 & 5 & 5 & 5 & 5 \\
\hline & \multirow{3}{*}{ C } & 80 & $2-3$ & 3 & 3 & 4 & 4 & 4 & 4 & 4 \\
\hline & & 95 & $3-4$ & 4 & 4 & 5 & 5 & $4-5$ & 4-5 & 4-5 \\
\hline & & 110 & 4 & 4 & 4-5 & 5 & 5 & 5 & 5 & 5 \\
\hline & \multirow{3}{*}{$\mathrm{D}$} & 80 & $2-3$ & 3 & 3 & 4 & 4 & 4 & 4 & 4 \\
\hline & & 95 & $3-4$ & 4 & 4 & 5 & 5 & $4-5$ & $4-5$ & 4-5 \\
\hline & & 110 & 4 & 4 & 4-5 & 5 & 5 & 5 & 5 & 5 \\
\hline
\end{tabular}

A: Disperse Red 60 and Reactive Red 124; B: Disperse Orange 96 and Reactive Orange 64; C: Disperse Blue 183 and Reactive Blue 116; D: Disperse Yellow 49 and Reactive Yellow 14.

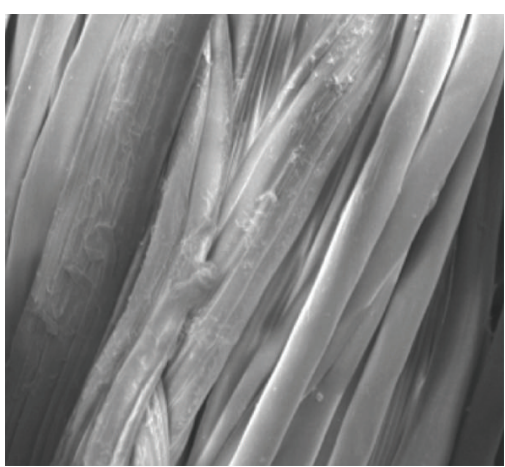

FIgURE 11: SEM photograph of Ac-MAc-nH-treated 80:20 PCF.

opening up of the structured assembly enhancing more dye uptake when compared with the untreated [35]. Increase in pretreatment duration causes much pronounced effect on the treated materials, which leads to improved dye uptake. The above observation is supported by the weight loss and

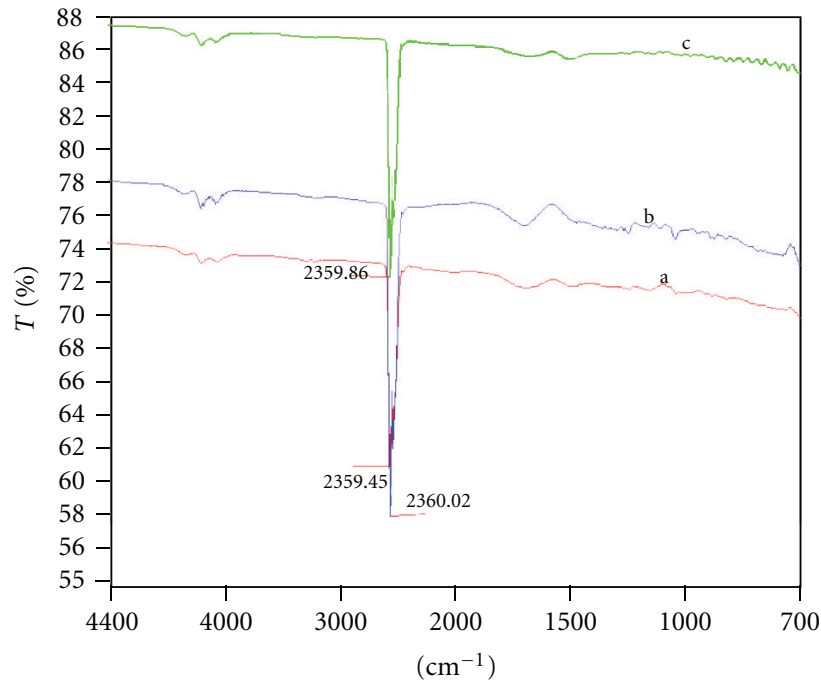

FIGURE 12: FTIR spectra of $80: 20$ PCF treated with Ac-EA-Cf for different durations; (a) untreated, (b) treated for 4 min and (c) treated for $30 \mathrm{~min}$. 
TABLE 5: Weight loss/abrasion resistance of treated and untreated $80: 20$ PCF.

\begin{tabular}{lccc}
\hline $\begin{array}{l}\text { Solvent } \\
\text { system }\end{array}$ & $\begin{array}{c}\text { Pretreatment } \\
\text { time (min.) }\end{array}$ & $\begin{array}{c}\text { Percentage loss } \\
(\%)\end{array}$ & $\begin{array}{c}\text { Abrasion resistance } \\
\text { no. cycles }\end{array}$ \\
\hline \multirow{4}{*}{ Ac-EA-Cf } & 2 & 0.098 & 7295 \\
& 4 & 0.257 & 7410 \\
& 6 & 0.750 & 7800 \\
& 10 & 0.820 & 7745 \\
& 20 & 0.980 & 7550 \\
& 30 & 1.120 & 7475 \\
Ac-MAc- & 2 & 1.350 & 7275 \\
nH & 4 & 0.050 & 7255 \\
& 8 & 0.130 & 7380 \\
& 10 & 0.475 & 7763 \\
& 20 & 0.650 & 7715 \\
& 30 & 0.780 & 7510 \\
Untreated & - & 0.987 & 7442 \\
\hline
\end{tabular}

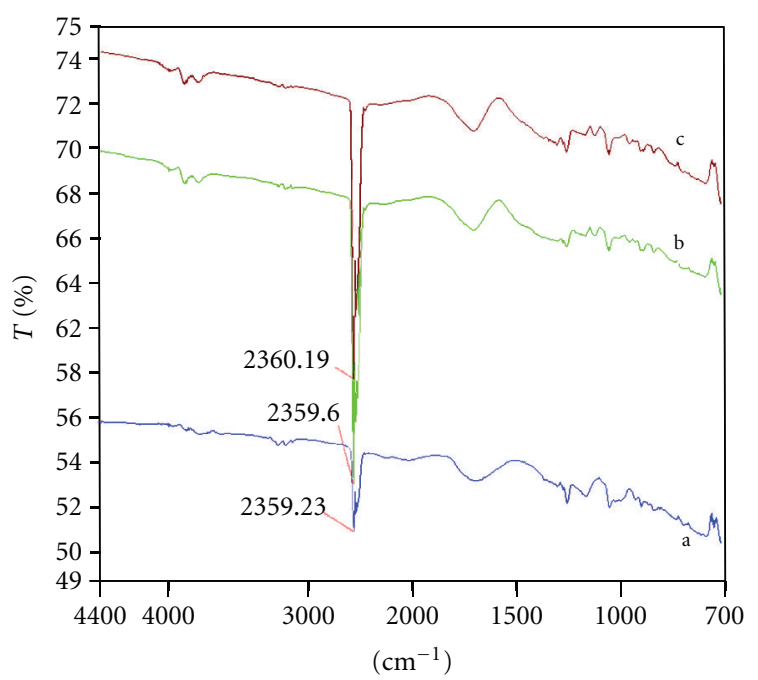

FIgURe 13: FTIR spectra of $80: 20$ PCF treated with Ac-MAc-nH for different durations; (a) untreated, (b) treated for 4 min and (c) treated for $30 \mathrm{~min}$.

tearing strength measurements wherein no much loss in weight and strength was observed.

\section{Conclusion}

The effect of azeotropic mixture of solvent pretreatments on the dyeing behavior of $80: 20$ PCF was studied. As the pretreatment time increased, the dye uptake was found to increase. The slight improvement in the fastness properties of the pretreated fabrics revealed that the treatment has not affected the dye-fibre bond and the improvement in

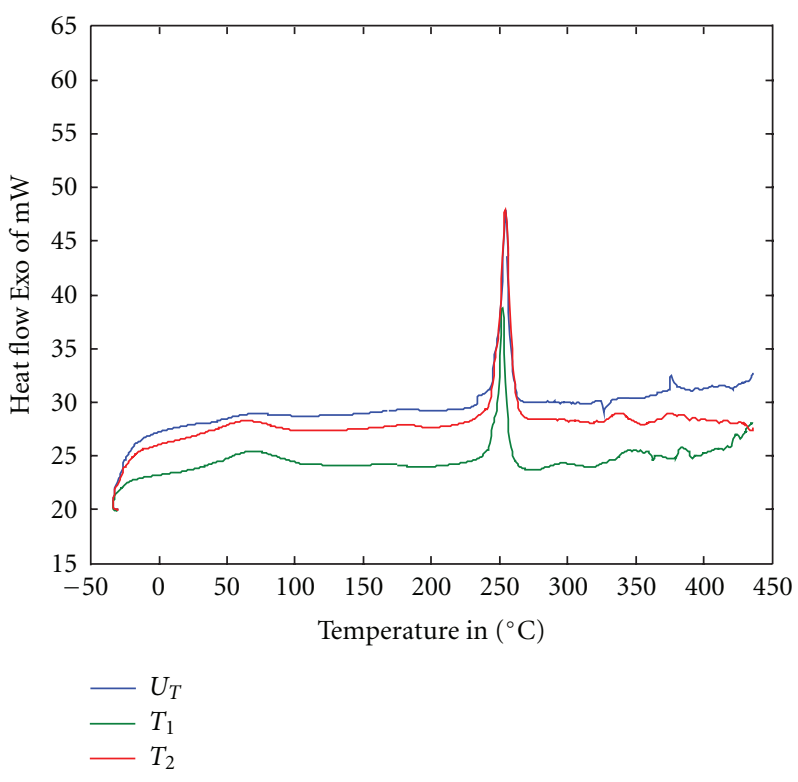

FIgURE 14: DSC of $80: 20$ PCF treated with Ac-EA-Cf for different durations; $\mathrm{UT}$ : untreated, $\mathrm{T}_{1}: 4 \mathrm{~min}$, and $\mathrm{T}_{2}: 30 \mathrm{~min}$.

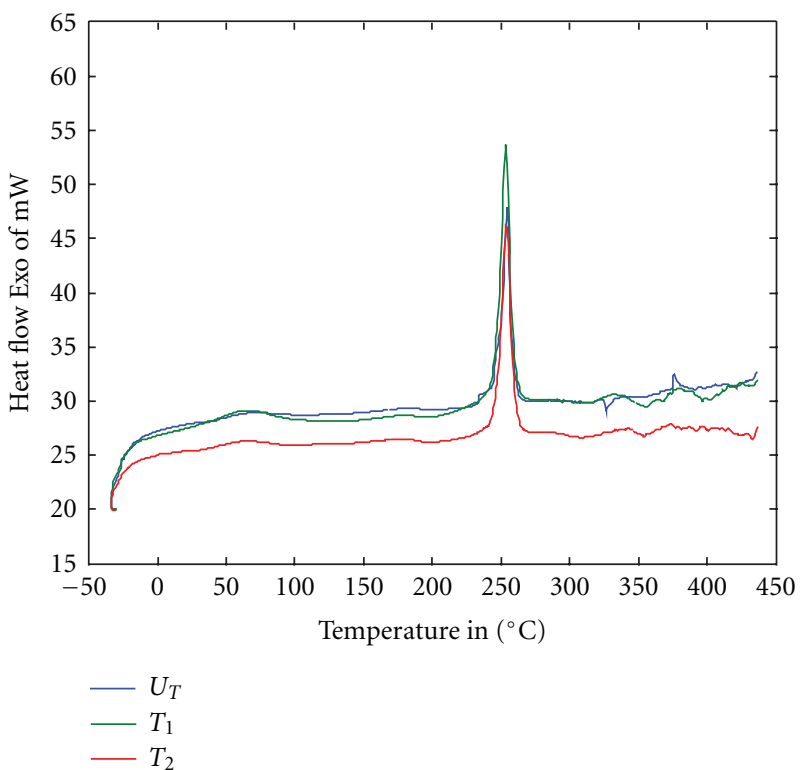

FIGURE 15: DSC of $80: 20$ PCF treated with Ac-MAc-nH for different durations; $\mathrm{UT}$ : untreated, $\mathrm{T}_{1}: 4 \mathrm{~min}$, and $\mathrm{T}_{2}: 30 \mathrm{~min}$.

fastness is due to improved dye pickup and dye-fibre bond formation. The abrasion resistance measurements of the treated materials show that there was a small increase in abrasion resistance of solvent pretreated samples up to 6 minutes pretreatment time. Prolonged solvent pretreatments led to decrease in abrasion resistance when treated for more than 6 minutes. As the time of pretreatment increased, the weight loss of the fabric was also found to increase. SEM studies showed that the azeotropic solvent mixtures attacked the entire surface of the fabric materials and caused erosion. As the time of solvent treatment increased, erosion 
TABLE 6: Thermal behavior of azeotropic solvent-mixture-treated $80: 20$ PCF.

\begin{tabular}{lcccccc}
\hline Solvent system & Pretreatment time $(\mathrm{min})$. & $T_{1}$ & $T_{0}$ & $T_{2}$ & $T_{m}$ & Max. heat flow in mW \\
\hline \multirow{2}{*}{ Ac-EA-Cf } & 4 & 233.2 & 243.4 & 270.3 & 254.6 & 46.22 \\
& 30 & 233.7 & 243.8 & 270.6 & 254.9 & 46.92 \\
Ac-MAc-nH & 4 & 233.8 & 242.1 & 270.5 & 254.4 & 44.97 \\
& 30 & 233.9 & 242.7 & 270.6 & 254.7 & 45.09 \\
& untreated & 233.1 & 240.6 & 270.4 & 254 & 36.55 \\
\hline
\end{tabular}

$T_{1}, T_{0}, T_{2}$, and $T_{m}$ correspond to starting, onset, final, and melting temperatures, respectively.

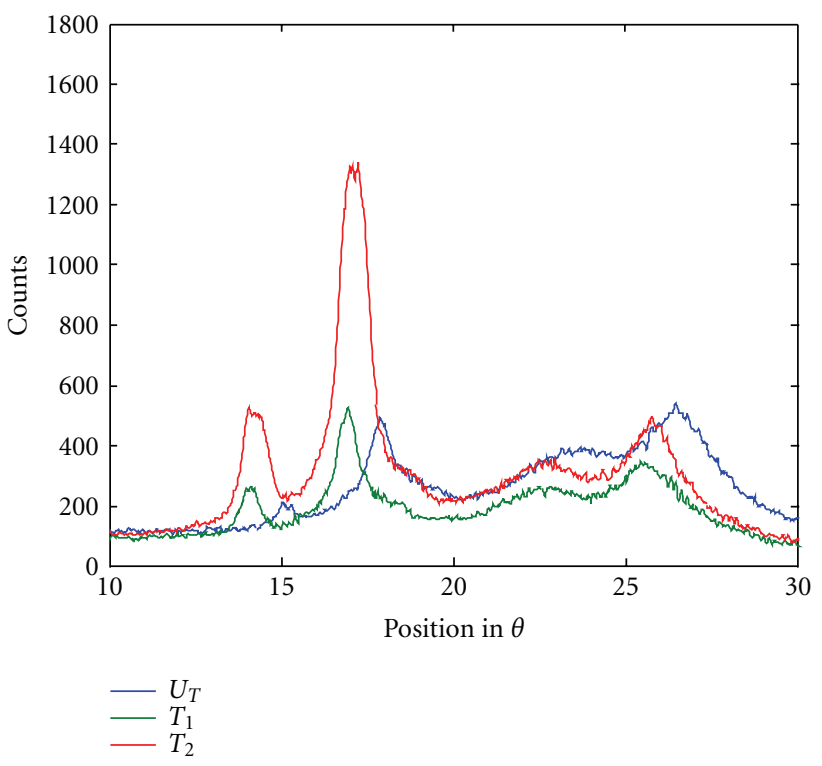

FIgURE 16: XRD of $80: 20$ PCF treated with Ac-EA-Cf for different durations; $\mathrm{UT}$ : untreated, $\mathrm{T}_{1}: 4 \mathrm{~min}$, and $\mathrm{T}_{2}: 30 \mathrm{~min}$.

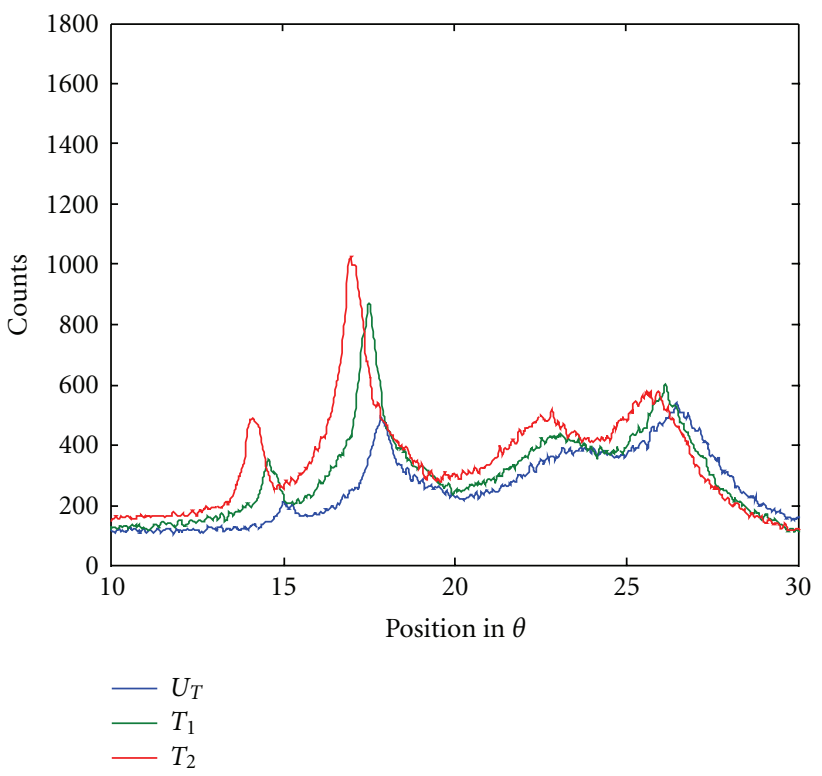

FIGURE 17: XRD of $80: 20$ PCF treated with Ac-MAc-nH for different durations, $\mathrm{UT}=$ untreated, $\mathrm{T}_{1}=4 \mathrm{~min}$. and $\mathrm{T}_{2}=30 \mathrm{~min}$. propagated into the fibre structure resulting in the formation of elongated pits or cavities on the surface. FTIR analysis of treated and untreated fabrics showed that there was no structural change or introduction of any functional groups or alteration of the existing groups in the case of solvent-treated materials used in the study. It is also concluded from the XRD and DSC analysis that the solvent treatment has disturbed the crystalline distribution probably by creating more cavity and pores resulting in opening up of the structured assembly. The improvement in the dye uptake of solvent-treated fabrics is due to large increase in intersurface area by swelling and greater segmental mobility of polymer molecules.

\section{References}

[1] R. J. Beech, Fibre-Reactive Dyes, Logos Press, London, UK, 1970.

[2] J. M. Cardamone and J. D. Turner, "Cationic applications for union dyeing wool/cotton blends," Textile Chemist and Colorist \& American Dyestuff Reporter, vol. 32, no. 6, pp. 49-53, 2000.

[3] S. D. Kim, J. L. Lee, C. H. Ahn, K. S. Kim, and K. S. Lee, "Dyeing of N/P union fabric with reactive disperse dyes," Journal of Korean Society of Dyers and Finishers, vol. 16, no. 1, pp. 26-33, 2004.

[4] S. N. Croft, D. M. Lewis, R. Orita, and T. Sugimoto, "Neutral-fixing reactive dyes for cotton. Part 1-synthesis and application of quaternized S-triazinyl reactive dyes," Journal of the Society of Dyers and Colourists, vol. 108, no. 4, pp. 195-199, 1992.

[5] T. Sugimoto, "Neutral-fixing reactive dyes for cotton. Part 2commercial reactive dyestuffs and their classification," Journal of the Society of Dyers and Colourists, vol. 108, no. 11, pp. 497500, 1992.

[6] J. Y. Kim, K. P. Lee, H. M. Park, M. S. Yoon, and K. H. Chao, "The one bath onestep dyeing of nylon/cotton blends with acid dyes and mononicotinic acid-triazine type reactive dyes," Journal of Korean Society of Dyers and Finishers, vol. 16, no. 5, pp. 1-7, 2004.

[7] B. Jin-Seok, H. P. Jong, K. Joonseok, and D. K. Sung, "Dyeing and fastness properties of a reactive disperse dye on PET, nylon, silk and N/P fabrics," Fibers and Polymers, vol. 7, no. 2, pp. 174-179, 2006.

[8] K. Mikyung, Y. Seokhan, K. Taekyeong, B. Jin-seok, and Y. Namsik, "Dyeing of cotton and polyester/cotton blend with disperse dyes using sodium 2-(2,3-dibromopropionylamino)5-(4,6-dichloro-1,3,5-triazinylamino)- benzenesulfonate," Fibers and Polymers, vol. 7, no. 4, pp. 352-357, 2006.

[9] Y. A. Youssef, Y. A. Nahed, A. A. Ahmed, A. A. Mousa, and M. E. Reda, "Alkaline dyeing of polyester and polyester/cotton 
blend fabrics using sodium edentate," Journal of Applied Polymer Science, vol. 108, no. 1, pp. 342-350, 2008.

[10] M. Shingo, K. Katsushi, H. Toshio, and M. Kenji, "One-bath dyeing of polyester/cotton blends with reactive disperse dyes in supercritical carbon dioxide," Textile Research Journal, vol. 74, no. 11, pp. 989-994, 2004.

[11] H. Najafi, R. Assefipour, M. Hajilari, and H. R. Movahed, "One bath method dyeing of polyester/cotton blend fabric with sulphatoethylsulphonyl disperse/reactive dyes treatment by chitin biopolymer," African Journal of Biotechnology, vol. 8, no. 6, pp. 1127-1135, 2009.

[12] J. W. Davis, "The preparation and dyeing of polyester-cotton fabrics," Journal of the Society of Dyers and Colourists, vol. 89, no. 3, pp. 77-80, 1973.

[13] B. Muralidharan, T. Mathanmohan, and J. Ethiraj, "Effect of acetonitrile pretreatment on the physicochemical behavior of $100 \%$ polyester fabric," Journal of Applied Polymer Science, vol. 91, no. 6, pp. 3871-3878, 2004.

[14] A. Bendak and W. M. Raslan, "Dyeability improvement of polyester pretreated with some alkoxides," Journal of Applied Polymer Science, vol. 108, no. 1, pp. 7-13, 2008.

[15] Dystar Textilfarben GMBH \& Co, Dyeing Polyester-Cotton Blend Fabrics, World Intellectual Property Organization, Geneva, Switzerland, 2009.

[16] G. Ryland, "Liquid mixrures of constant boiling point," Journal of the American Chemical Society, vol. 22, p. 384, 1899.

[17] M. Lecat, La Tension de Vapeur des Melanges de Liquides L'Azeotropisme, Lamartin, Bruxelles, Belgium, 1918.

[18] W. Bernhard, "Equilibrium melting of flexible linear macromolecules," Polymer Engineering \& Science, vol. 18, no. 6, pp. 431-436, 1978.

[19] M. R. Wafaa, B. Ahmed, M. K. Eid, and F. Thanaa, "Modification of polyester fabric by chemical/thermal treatment to improve dyeing ability," Coloration Technology, vol. 126, no. 4, pp. 231-236, 2010.

[20] S. R. Arthur, W. Hans-Dietrich, and R. Ludwig, "Interactions of nonaqueous solvents with textile fibers," Textile Research Journal, vol. 43, no. 3, pp. 176-183, 1976.

[21] D. Jayshree, R. Kumar, and H. C. Srivastava, "Studies on modification of polyester fabrics 1: alkaline hydrolysis," Journal of Applied Polymer Science, vol. 33, no. 2, pp. 455-477, 1987.

[22] H. D. Weigmann and A. S. Ribnick, "Interactions of nonaqueous solvents with textile fibers: part IV: effects of solvents on the mechanical properties of various textile yarns," Textile Research Journal, vol. 44, no. 3, pp. 165-173, 1974.

[23] A. S. Ribnick, H. D. Weigmann, and L. Rebenfeld, "Interactions of nonaqueous solvents with textile fibers: part I: effect of solvents on the mechanical properties of a polyester yarn," Textile Research Journal, vol. 42, no. 12, pp. 720-726, 1972.

[24] J. L. Williams and A. Peterlin, "A study of sorption and permeability of gases and vapours in solvent-drawn polyethylene," Die Makromolekulare Chemie, vol. 135, no. 1, pp. 41-47, 1970.

[25] S. Rajendran, S. S. Ramasamy, and S. P. Mishra, "Studies on dyeing and structural behavior of chemically treated polyester yarns," Journal of Applied Polymer Science, vol. 62, no. 7, pp. 989-1001, 1996.

[26] H. W. Siesler, "Fourier transform infra red characterization of polymers," in Fourier Transform-Infra Red Characterization of Polymers (Polymer Science and Technology, V. 36), H. Ishida, Ed., Plenum Press, New York, NY, USA, 1987.

[27] A. T. Debra and H. You-Lo, "Solvent-induced structural changes in sulfonated poly(ethylene terephthalate) (SPET) fibers," Journal of Applied Polymer Science, vol. 51, no. 7, pp. 1291-1301, 1994.
[28] D. Chidambaram, R. Venkatraj, and P. Manisankar, "Solventinduced modifications in polyester yarns. II. Structural and thermal behavior," Journal of Applied Polymer Science, vol. 89, no. 6, pp. 1555-1566, 2003.

[29] J. Hasan, W. Joshua, and R. Ludwig, "The effects of orientation and crystallinity on the solvent-induced crystallization of poly(ethylene terephthalate). 1. Sorption- and diffusionrelated phenomena," Journal of Applied Polymer Science, vol. 26, no. 6, pp. 1795-1811, 1981.

[30] S. Rajendran and S. P. Mishra, "Chemical, structural and thermal changes in PET caused by solvent induced polymer crystallisation," Polymers and Polymer Composites, vol. 15, no. 2, pp. 103-110, 2007.

[31] A. K. Kulshreshtha, A. H. Khan, and G. L. Madan, "Xray diffraction study of solvent-induced crystallization in polyester filaments," Polymer, vol. 19, no. 7, pp. 819-823, 1978.

[32] B. H. Knox, H. D. Weigmann, M. G. Scott, and L. Rebenfeld, "The Effects of an aqueous medium on the structure and physical properties of a polyester yarn," Textile Research Journal, vol. 51, no. 8, pp. 549-558, 1981.

[33] L. Vicini, A. Seves, M. Canetti, P. Sadocco, and R. Galli, "Influence of an aqueous acetone medium on the thermal behaviour of solvent treated poly(ethylene terephthalate)," Polymer, vol. 25, no. 12, pp. 1774-1778, 1984.

[34] H. D. Weigmann, "Interaction between fibres and organic solvents," in Handbook of Fiber Science and Technology Volume 1: Chemical Processing of Fabrics: Fundamentals and Preparation, Part A, M. Lewin and S. B. Sello, Eds., pp. 1-49, Marcel Dekker, New York, NY, USA, 1983.

[35] P. M. Stephen, R. Ludwig, and H. D. Weigmann, "Interactions of nonaqueous solvents with textile fibres: part XI. Nomex shrinkage behavior," Textile Research Journal, vol. 51, no. 5, pp. 323-331, 1981. 

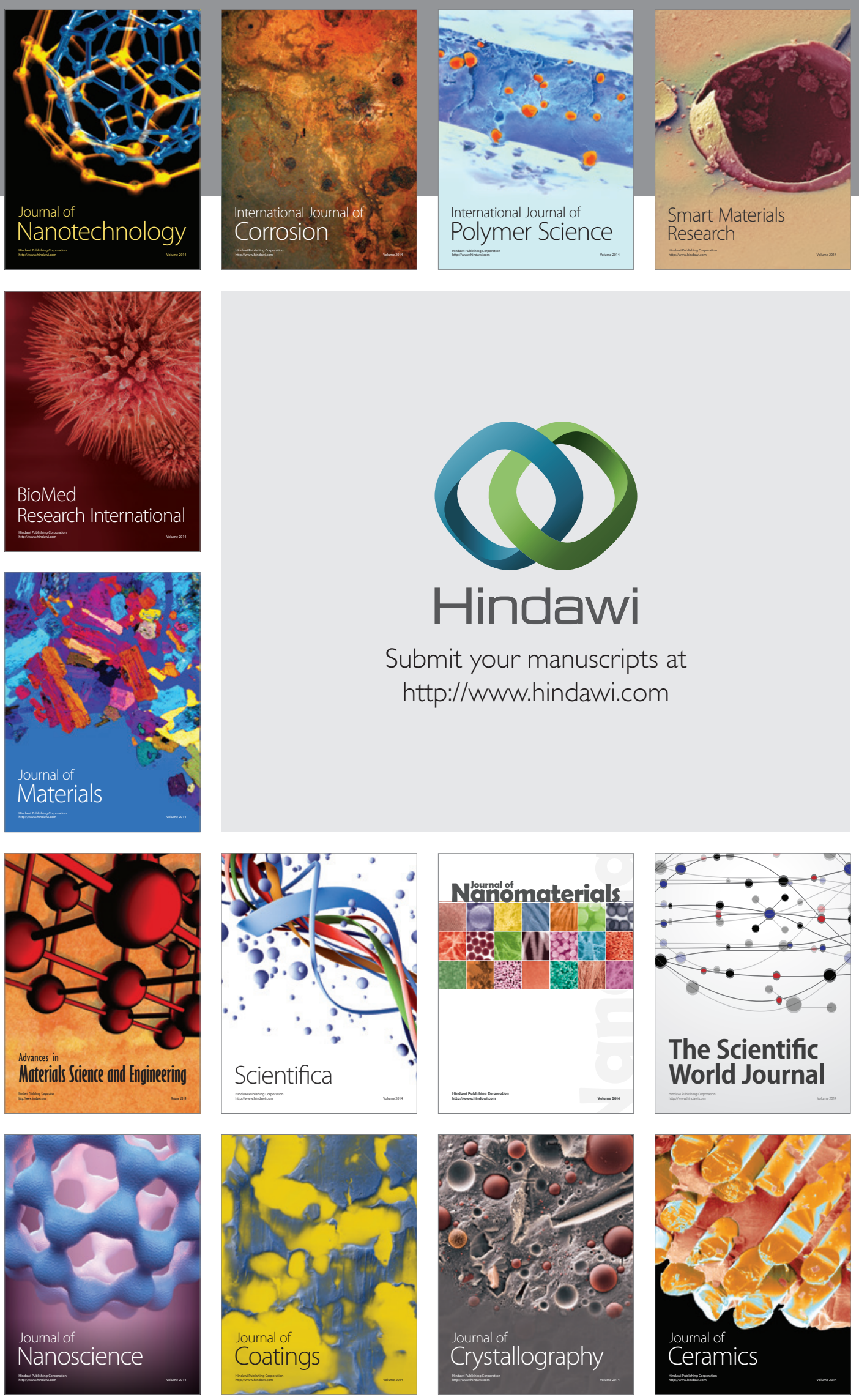

The Scientific World Journal

Submit your manuscripts at

http://www.hindawi.com

\section{World Journal}

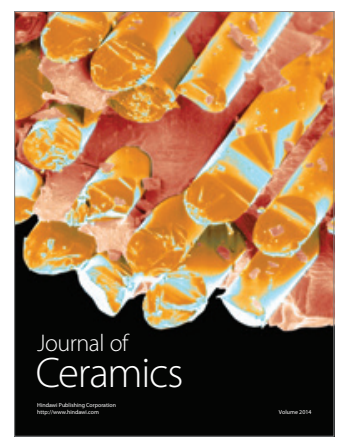

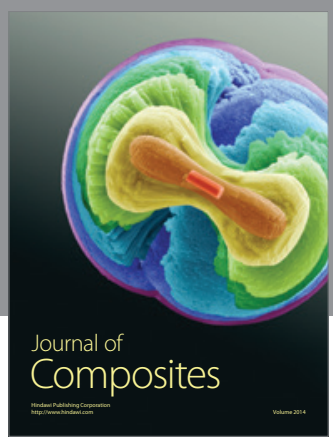
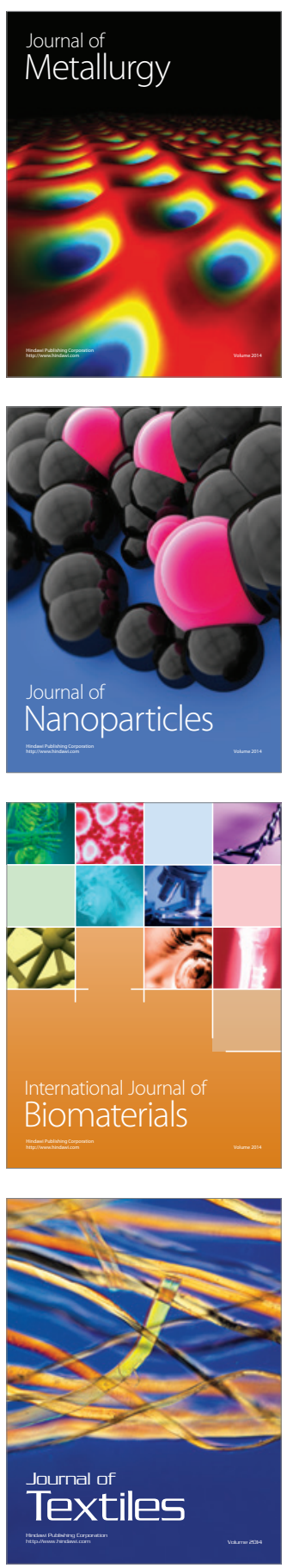